\title{
Angular Momentum Transport and Dynamo Effect in Kepler Disks
}

\author{
Günther Rüdiger, Udo Ziegler \\ Astrophysikalisches Institut Potsdam, \\ An der Sternwarte 16, D-14482 Potsdam, Germany
}

\begin{abstract}
Properties have been demonstrated of the magneto-rotational instability for two different applications, i.e. for a global spherical model and a box simulation with Keplerian background shear flow. In both nonlinear cases a dynamo operates with a negative (positive) $\alpha$-effect in the northern (southern) disk hemisphere and in both cases the angular momentum transport is outwards. Keplerian accretion disks should therefore exhibit large-scale magnetic fields with a dipolar geometry of the poloidal components favoring jet formation.
\end{abstract}

\section{Angular Momentum Transport}

The problem of angular momentum transport during star formation probably cannot be solved by companion formation alone. After all some single stars exist and disk accretion must occur. Accretion in disks requires a highly effective instability transporting the angular momentum outwards and mass inwards. This is not trivially fulfilled as pure hydrodynamical simulations show. But it turns out to be much easier if magnetic fields are involved.

There is also another issue. Almost all accretion disks show the jet phenomenon. In order to accelerate and to collimate the jet, a dipolar large-scale field is most suitable. All the dynamo simulations, however, yield quadrupolar field geometry if the dynamo alpha is positive in the upper disk plane (and negative in the lower disk plane). We therefore need a turbulent dynamo which provides the opposite sign for the dynamo alpha. This appears impossible in dynamo theories based on the convective instability (v. Rekowski et al. 2000).

The definitions are simple. The conservation law of angular momentum is $\operatorname{div} \rho r \mathcal{T}=0$ with the main transporters as

$$
\mathcal{T}=\left\langle u_{\phi}\right\rangle\langle\boldsymbol{u}\rangle-\frac{1}{\mu_{0} \rho}\left\langle B_{\phi}\right\rangle\langle\boldsymbol{B}\rangle+\left\langle u_{\phi}^{\prime} \boldsymbol{u}^{\prime}\right\rangle-\frac{1}{\mu_{0} \rho}\left\langle B_{\phi}^{\prime} \boldsymbol{B}\right\rangle
$$

written in the usual notation for flow $\boldsymbol{u}$ and magnetic field $\boldsymbol{B}$. The angular momentum transport due to turbulence is given by the last two terms. It is normalized with the local speed of sound $\left(c_{\mathrm{s}}\right)$, hence

$$
\mathcal{T}_{r \phi}^{\text {turb }}=\alpha_{\mathrm{SS}} c_{\mathrm{s}}^{2} \text {. }
$$

On the other hand, the correlation of flow and field form a turbulent electromotive force $\left\langle\boldsymbol{u}^{\prime} \times \boldsymbol{B}^{\prime}\right\rangle$ which, normalized with the vector of a dynamo-excited 

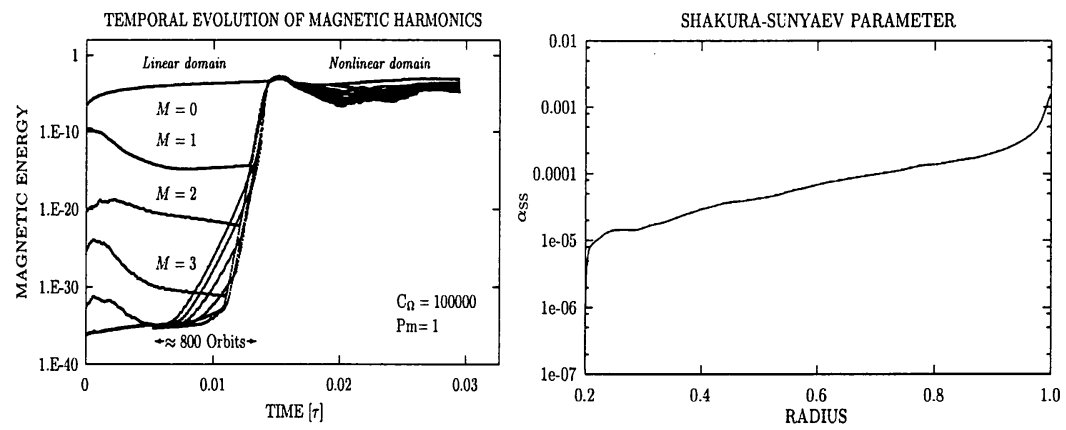

Figure 1. LEFT: Magnetic energy as a function of time. Several temporal stages can be distinguished: pure axisymmetric growth, exponential growth of the higher harmonics, and saturation in the nonlinear regime where a turbulent cascade has formed. RIGHT: The parameter $\alpha_{\mathrm{SS}}>0$ of angular momentum transport in the shear flow dynamo (cf. Drecker et al. 2000).

large-scale magnetic field, yields the dynamo- $\alpha$, i.e.

$$
\mathcal{E} \equiv\left\langle\boldsymbol{u}^{\prime} \times \boldsymbol{B}^{\prime}\right\rangle=\alpha_{\mathrm{dyn}} \circ\langle\boldsymbol{B}\rangle
$$

or, more simply, $\mathcal{E}_{\phi}=\alpha_{\phi \phi}\left\langle B_{\phi}\right\rangle$. Turbulent convection in plane-stratified atmospheres leads (in the northern hemisphere) to a positive $\alpha$-effect. The resulting angular momentum transport, however, is inwards. Therefore, some other mechanism must be operating in accretion disks.

\section{Shear Flow Dynamo of a Differentially Rotating Sphere}

The situation is different in a global MHD simulation of a full sphere embedded in vacuum and rotating with a rotation law in accord to $\Omega \propto s^{-q}$, where $s=r \sin \theta$ is the distance from the axis. $q$ is a free parameter. The results presented here are for a Kepler disk, $q=1.5$. In Kitchatinov \& Rüdiger (1997) we have shown with a linear analysis how all rotation laws with $0<q \leq 2$ lead to an unstable configuration if the spheres are threaded by a uniform magnetic field parallel to the rotation axis. In Drecker et al. (2000) the nonlinear equations

$$
\begin{aligned}
\frac{\partial \boldsymbol{u}}{\partial t} & +(\boldsymbol{u} \cdot \nabla) \boldsymbol{u}+\frac{1}{\rho} \nabla p=\nu \Delta \boldsymbol{u}+\frac{1}{\rho} \boldsymbol{j} \times \boldsymbol{B}, \\
\frac{\partial \boldsymbol{B}}{\partial t} & =\operatorname{curl}(\boldsymbol{u} \times \boldsymbol{B})+\eta \Delta \boldsymbol{B}
\end{aligned}
$$

with $\operatorname{div} \boldsymbol{u}=\operatorname{div} \boldsymbol{B}=0$ have been solved for a gaseous sphere with differential rotation and finite electrical resistivity. Of particular interest is the (dynamo-)case without imprinted external field presented in this contribution. Fig. 1 (LEFT) shows the result of the nonlinear simulations. At the beginning only the toroidal field is amplified by the action of differential rotation. Then the beginning of the saturation phase can be observed with a strong increase of poloidal magnetic 

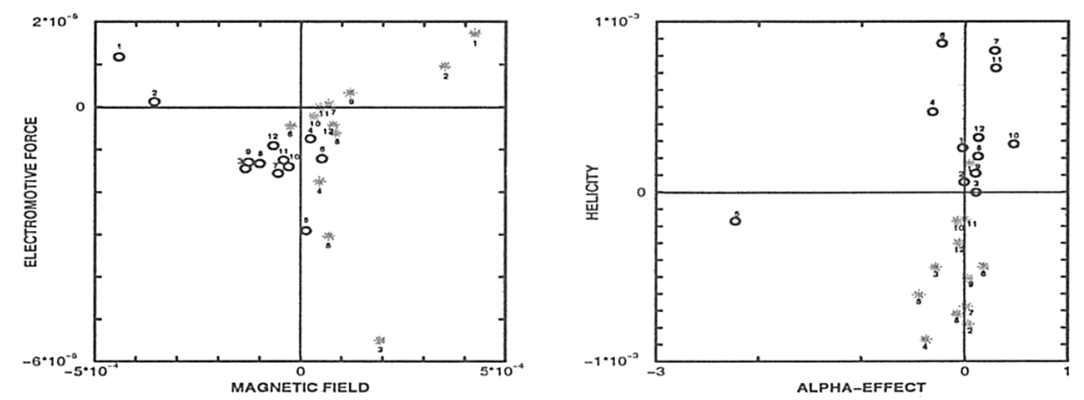

Figure 2. LEFT: The relation between the turbulent electromotive force and the mean magnetic field for the two hemispheres (asterix: north, circle: south). The time evolution is denoted by numbers. The dynamo $\alpha$-effect is negative (positive) in the northern (southern) hemisphere. RIGHT: The relation between helicity and dynamo $\alpha$-effect. The helicity is negative (positive) in the northern (southern) hemisphere.

components. Fig. 1 (RIGHT) shows the Shakura-Sunyaev 'viscosity- $\alpha$ ' (equ. (2)) to be positive, i.e. the angular momentum transport is indeed outwards. In order to find the sign of the dynamo- $\alpha$ in (equ. (3)), in Fig. 2 (LEFT) we plotted for several timesteps the relation between the turbulent EMF and the mean magnetic field $\left\langle B_{\phi}\right\rangle$. The two hemispheres are denoted differently. For almost all time steps the EMF is negative but the majority of the magnetic field amplitudes in the north (south) is positive (negative). The dynamo $\alpha$-effect is thus negative (positive) in the northern (southern) hemisphere. Hence, we have shown with global simulations that the magneto-rotational instability of shear flows can provide simultaneously positive viscosity- $\alpha$ and negative dynamo- $\alpha$. The relation between the $\alpha$-effect and helicity of the flow is shown in Fig. 2 (RIGHT). In both half-spheres the $\alpha$-parameter and helicity have the same sign in contrast to kinematic dynamo theory which predicts opposite signs between $\alpha$-effect and helicity.

\section{Local Simulations of a Differentially Rotating Keplerian Disk}

Using the shearing box approximation, local simulations of the nonlinear evolution of the magnetic shear instability in stratified disks yield that angular momentum transport is directed outwards. The average value is of the order of $\alpha_{\mathrm{SS}} \approx 10^{-2}$ measured in the saturation state into which the turbulent disk quickly settles after an initial exponential growth (Ziegler \& Rüdiger 2000). Angular momentum transport is mainly mediated by Maxwell stress rather than Reynolds stress; a clear indication that the flow is magnetically dominated. The time history of $\alpha_{\mathrm{SS}}$ is shown in more detail in Fig. 3.

The models with initial zero net magnetic flux show, despite the presence of substantial (numerical) dissipation, not only the amplification and maintainence of the magnetic field but, additionally, the development of a mean azimuthal field 


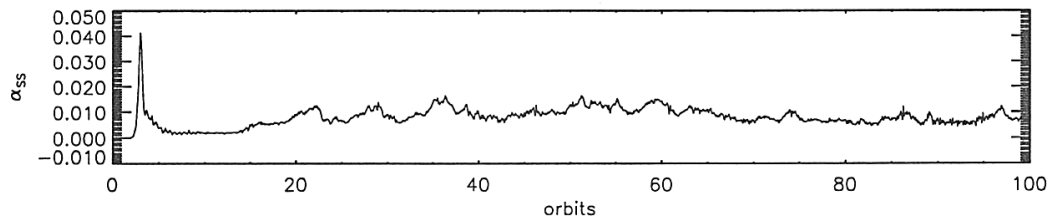

Figure 3. Time history of the Shakura-Sunyaev parameter $\alpha_{\mathrm{SS}}$.
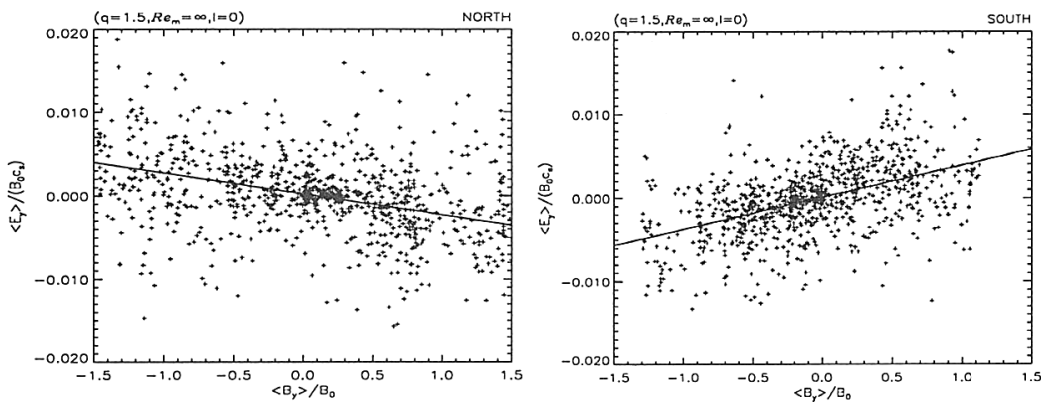

Figure 4. Dynamo $\alpha$-effect: relation between the mean turbulent EMF and mean azimuthal field in the northern (left) and southern (right) disk hemisphere for a large number of snapshots.

within the disk. A relation exists between the mean field and mean turbulent EMF illustrated in Fig. 4 for a large number of time snapshots. The northern and southern disk hemisphere is shown separately. The correlation turns out to be rather noisy. On average, however, $\alpha$ is negative in the northern part and positive in the southern part of the disk. Thus, the local simulations fully support the picture previously found in the global sphere simulations: accretion disk dynamos driven by the magnetic shear instability can provide both positive $\alpha_{\mathrm{SS}}$ and negative (positive) dynamo- $\alpha$ in the northern (southern) disk hemisphere.

\section{References}

Drecker, A., Rüdiger, G., \& Hollerbach, R. 2000, MNRAS, 317, 45

Kitchatinov, L. L., \& Rüdiger, G. 1997, MNRAS, 286, 757

v. Rekowski, M., Rüdiger, G., \& Elstner, D. 2000, A\&A, 353, 813

Ziegler, U., \& Rüdiger, G. 2000, A\&A, 356, 1141 

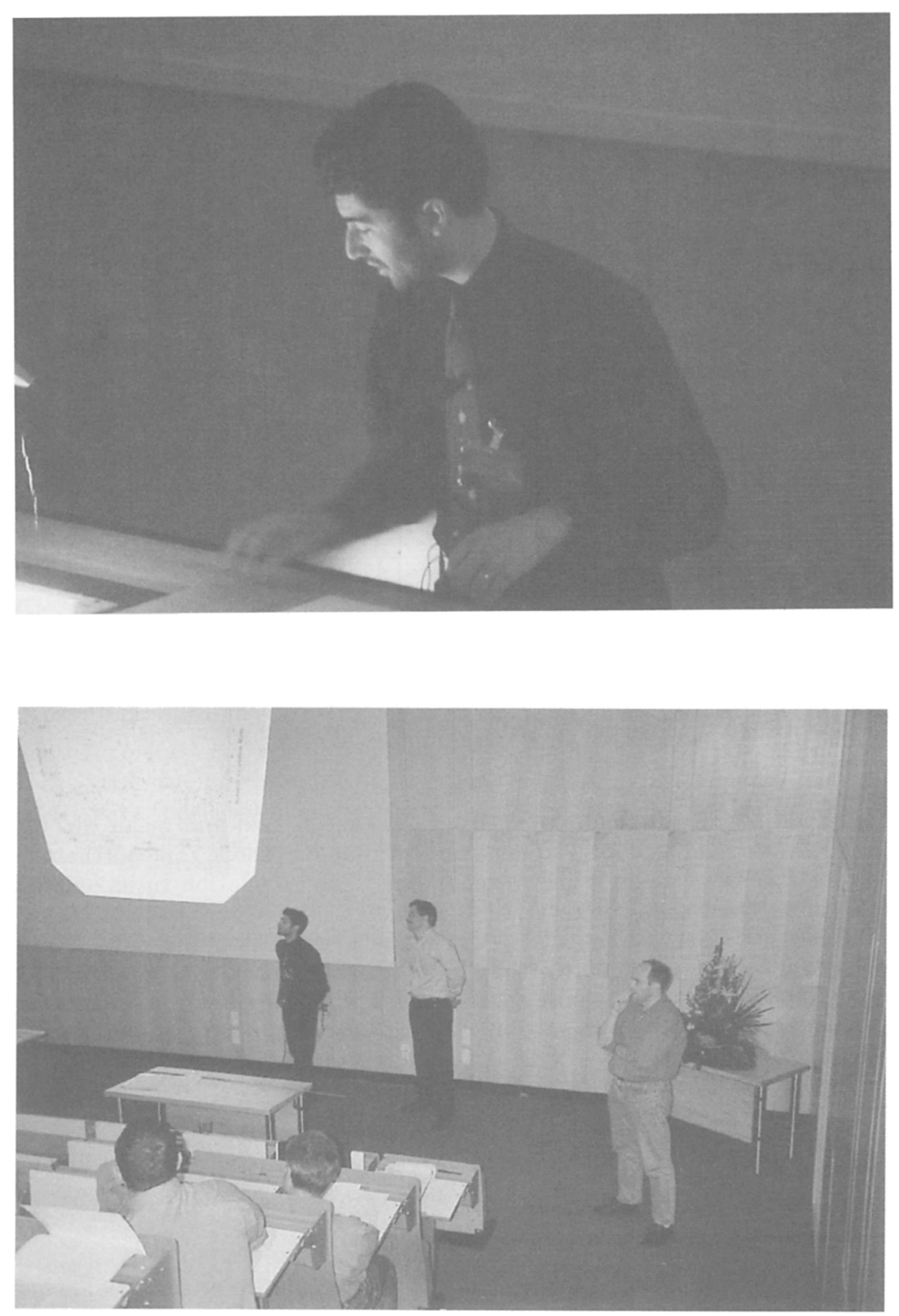

Keivan Stassun during his talk and during the discussion following his talk (with co-author Bob Mathieu and chairman Mark McCaughrean) 